\title{
Sensitive Polarimetric Search for Relativity Violations in Gamma-Ray Bursts
}

\author{
V. Alan Kostelecký ${ }^{1}$ and Matthew Mewes ${ }^{2}$ \\ ${ }^{1}$ Physics Department, Indiana University, Bloomington, Indiana 47405, USA \\ ${ }^{2}$ Physics Department, Marquette University, Milwaukee, Wisconsin 53201, USA
}

(Received 13 June 2006; published 2 October 2006)

\begin{abstract}
We show that the recent measurements of linear polarization in gamma rays from GRB 930131 and GRB 960924 constrain certain types of relativity violations in photons to less than parts in $10^{37}$, representing an improvement in sensitivity by a factor of 100000 .
\end{abstract}

PACS numbers: 03.30.+p, 11.30.Cp, 95.75.Hi, 98.70.Rz

At the fundamental level, nature is well described by the standard model of particle physics and Einstein's general relativity. The self-consistency and aesthetics of these theories suggest that they coalesce into a single underlying unified theory at extreme high energies. Experimental evidence of this notion is difficult to obtain because the energies involved are inaccessible. However, minuscule effects of the coalescence might be detectable in suitably sensitive experiments.

A promising candidate effect is relativity violations, which are associated with the breaking of Lorentz symmetry, the invariance of the laws of physics under rotations and boosts [1]. Here we show that the recent measurement of linear polarization in gamma rays from the gamma-ray bursts GRB 930131 and GRB 960924 [2] places a conservative bound on some types of relativity violations in photons at the level of $10^{-37}$. This sensitivity improves by a factor of 100000 over previous analyses [3], which used comparatively low-energy photons in the infrared to ultraviolet range, and it places strong limits on relativityviolating models.

The effects of Lorentz violations at attainable energies are determined by a theoretical framework known as the standard-model extension (SME), which describes general violations of Lorentz symmetry independent of their origin $[4,5]$. The SME provides a basis for experimental searches for relativity violations in many different arenas, including modern Michelson-Morley and Kennedy-Thorndike tests highly sensitive to the properties of light [6-9]. In general, light is composed of two independently propagating constituent waves, each possessing a polarization and a velocity. The SME predicts that certain forms of relativity violations cause light to experience birefringence, a change in polarization as it propagates in otherwise empty space. The changes grow linearly with distance traveled, so birefringence over cosmological scales offers an exceptionally sensitive signal for relativity violations. Searches for this vacuum birefringence using near-optical polarized light emitted from galaxies at cosmological distances yield some of the sharpest existing tests of relativity [3,10].

The SME coefficients that control Lorentz violation can be ordered according to their dimensionality, which offers a simple measure of their expected size [11]. Among the dominant terms in the photon sector of the SME are ten that induce vacuum birefringence of light. They predict changes in polarization that are reciprocally related to wavelength, so using higher-energy photons increases sensitivity to the effects. The corresponding ten coefficients controlling the birefringence are conveniently collected in matrices $\tilde{\kappa}_{e+}$ and $\tilde{\kappa}_{o-}$. Their magnitudes are currently bounded at the level of $10^{-32}$ by observations of polarized light from galaxies at cosmological distances (distances $L \sim 1 \mathrm{Gpc} \sim 10^{25} \mathrm{~m}$ ) in the near-optical range (energies $E \sim 1 \mathrm{eV}$ ) [3]. These sensitivities at present are roughly comparable to the best attained values in matter-based relativity tests involving first-generation particles [1215] and exceed present results from interferometric studies with second- and third-generation particles [16,17]. However, a gamma-ray burst (GRB) contains photons in the $\mathrm{MeV}$ range, so GRB polarimetry offers the potential for a millionfold improvement in sensitivity assuming similar propagation distances.

GRB polarimetry is presently in its infancy [2,18-25]. Some time ago, evidence was offered for a high degree of linear polarization $\Pi$ in emissions from GRB 021206 extending into the $\mathrm{MeV}$ range [18]. The claimed value $\Pi_{021206}=(80 \pm 20) \%$ was based on a study of detector scatterings of gamma-ray photons in the Reuven Ramaty High Energy Solar Spectroscopic Imager (RHESSI). This result has proved controversial [19-21] but served to stimulate interest in the subject. Recently, nonzero linear polarizations of $\Pi_{930131}>35 \%$ in GRB 930131 and of $\Pi_{960924}>50 \%$ in GRB 960924 have been demonstrated [2]. These latter results are based on an analysis of emissions at energies up to $100 \mathrm{keV}$, recorded by the Burst and Transient Source Experiment via the scattering of gammaray photons off the Earth's atmosphere.

Our goal here is to consider the implications of these observations for the dominant relativity violations in the SME, controlled by the coefficients $\tilde{\kappa}_{e+}$ and $\tilde{\kappa}_{o-}$. We disregard possible gravitational couplings $[5,26]$ and assume that the coefficients $\tilde{\kappa}_{e+}$ and $\tilde{\kappa}_{o_{-}}$are constant in spacetime. If these coefficients originate in spontaneous Lorentz violation [27], they have companion NambuGoldstone fluctuations that might be interpreted as the photon [28] or graviton [29] fields, but this is a secondary 
issue and is disregarded in this work. Subdominant birefringent effects, which grow quadratically or faster in energy and correspond to nonrenormalizable terms in the SME, have been considered for GRB 021206 in the context of specific Lorentz-violating scenarios using modified dispersion relations [30,31]. Other dominant birefringent effects in the SME also exist $[4,10]$, but they are energy independent and so cannot benefit from the higher photon energies accessible in gamma-ray bursts.

Nonzero values of the coefficients $\tilde{\kappa}_{e+}$ and $\tilde{\kappa}_{o-}$ modify the usual photon phase velocity to the form $v_{ \pm} / c=1+$ $\rho \pm \sigma$, where $\rho$ and $\sigma$ are source-dependent combinations of $\tilde{\kappa}_{e+}$ and $\tilde{\kappa}_{o-}$ [4]. The two choices of sign, $v_{+}$and $v_{-}$, correspond to the velocities of the two constituent birefringent waves, which have linear and mutually orthogonal polarizations. The difference in phase velocities induces a phase shift $\Delta \phi$ between the two component waves, given by

$$
\Delta \phi \simeq 2 \sigma L_{\mathrm{eff}} E,
$$

where $E$ is the photon energy and $L_{\text {eff }}$ is the effective light travel distance, including corrections stemming from the expansion of the Universe during the propagation [3]. We see that $\Delta \phi$ depends linearly on the combination $\sigma$ of coefficients for relativity violations. In contrast, the combination $\rho$ affects both wave components equally and so induces no birefringence.

The interference between the two birefringent components causes the polarization of light to vary as $\Delta \phi$ changes. Through Eq. (1), the polarization becomes dependent both on the effective distance $L_{\text {eff }}$ and on the photon energy $E$. Larger values of $L_{\text {eff }}$ and $E$ therefore result in increased sensitivity to the relativity-violating quantity $\sigma$.

An elegant visualization of the effects of $\sigma$ on the polarization of light is offered by the notion of the Stokes vector and the Poincaré sphere [3]. The Stokes vector $\vec{s}$ consists of three numbers $\vec{s}=\left(s_{1}, s_{2}, s_{3}\right)$ forming a threedimensional vector in the abstract Stokes-parameter space. For coherent light, $\vec{s}$ uniquely determines the relative degree of linear and circular polarization, the polarization angle $\psi$, and the helicity.

Figure 1 illustrates the connection between the Stokes vector and the standard elliptical-polarization angles $\psi$ and $\chi$. For the usual polarization ellipse, $\psi$ characterizes the orientation, while $\chi$ characterizes the eccentricity and the helicity. For the Poincaré sphere, each surface point represents a unique polarization and corresponds to the tip of a unit-normalized Stokes vector. Pure-linear polarizations lie on the equator with $\chi=0$, while right- and left-handed circular polarizations are represented by the north pole $\chi=+45^{\circ}$ and the south pole $\chi=-45^{\circ}$, respectively. Arbitrary right- and left-handed elliptical polarizations correspond to points on the northern and southern hemispheres.

As the light propagates in the presence of relativity violations, the change in polarization corresponds to a

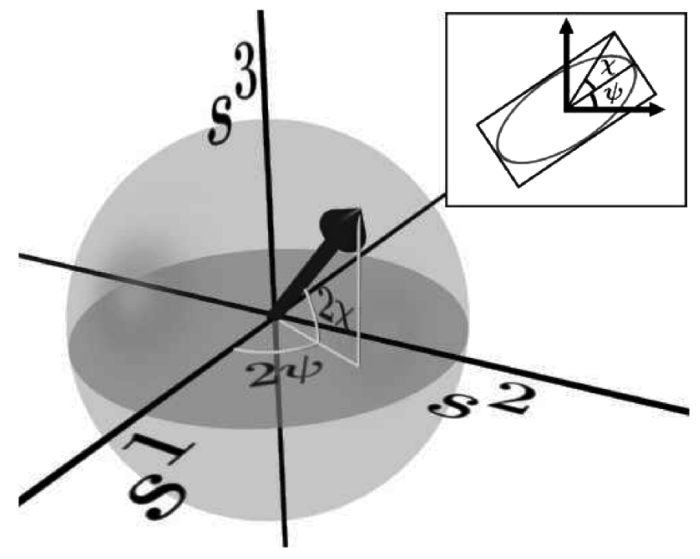

FIG. 1. Elliptical polarization and the Poincaré sphere.

change in orientation of the Stokes vector. The tip of the Stokes vector therefore follows a path on the Poincaré sphere. Given nonzero values of $\tilde{\kappa}_{e+}$ and $\tilde{\kappa}_{o_{-}}$, this path is obtained by rotating the Stokes vector through the angle $\Delta \phi$ about an axis $\vec{s}_{+}$. The axis $\vec{s}_{+}$turns out to be just the Stokes vector for the $v_{+}$component of the light alone. Since this component is linearly polarized, its Stokes vector $\vec{s}_{+}$lies in the $s_{1}-s_{2}$ plane. The Stokes vector $\vec{s}_{-}$for the $v_{-}$component points in the opposite direction, $\vec{s}_{-}=-\vec{s}_{+}$.

For a fixed photon energy $E$ and effective travel distance $L_{\text {eff }}$, Eq. (1) shows that the magnitude $\Delta \phi$ of the rotation, and hence the size of the effect, is controlled by the relativity-violating quantity $\sigma$. The orientation $\vec{s}_{+}$of the rotation axis also plays a role. It can be parametrized by a single source-dependent angle $\xi$, which is the angle between $\vec{s}_{+}$and the $s^{1}$ axis. For a given source, it follows that birefringence and the subsequent change in polarization are then completely determined by $\sigma$ and $\xi$.

In applying these results to measurements of GRB polarization, the key idea is that a nonzero $\sigma$ causes the polarization to change rapidly with energy at some large effective distance $L_{\text {eff }}$. The observational signal is a reduced measured degree of linear polarization $\Pi$. Two effects contribute to the reduction. One is an energydependent mixing from linear to circular polarization, corresponding to a rotation of the Stokes vector away from the equator of the Poincare sphere. The second is an energy-dependent change in the polarization angle $\psi$. This arises because as $\vec{s}$ rotates about $\vec{s}_{+}$the angle $\psi$ oscillates about a mean value of $\xi / 2$ or $(\xi+\pi) / 2$. Both of these effects result in a loss of coherence over a range of $E$, thereby reducing the measured value of $\Pi$.

To get conservative bounds on Lorentz violation, we assume the emissions associated with GRB 930131, GRB 960924, and GRB 021206 initially have 100\% linear polarization at a constant polarization angle $\psi_{0}$ over the relevant energy range (31-98 keV for GRB 930131 and GRB 960924 [2] and 150-2000 keV for GRB 021206 [18]). Using simulations for the photons and an appropriate smearing over the relevant energy range, we then calculate the effective degree of linear polarization $\Pi_{\text {eff }}$ and the 
effective polarization angle $\psi_{\text {eff }}$ seen at the Earth, as a function of the relativity-violating parameters $\sigma$ and $\xi$.

For the simulations, the effective distance $L_{\text {eff }}$ to each GRB is required. This includes a measure of the increase in photon wavelength resulting from the cosmological expansion. For a universe with equation of state $p / \rho=w$ constant, the effective distance is then $L_{\text {eff }}=$ $2\left[1-(1+z)^{-(1+3 w) / 2}\right] /(1+3 w) H_{0}$. At small redshifts, this is independent of $w: L_{\text {eff }}=z / H_{0}$. In what follows, we use this result with $H_{0}=71 \mathrm{~km} \mathrm{~s}^{-1} \mathrm{Mpc}^{-1}$.

Reliable GRB redshifts are typically obtained through observations of their optical counterparts. For GRB 021206, we take $z=0.3[32,33]$. No direct measurement is available for GRB 930131 or GRB 960924, but there are several attempts at distance determinations from gamma-ray spectral data alone [33-36]. For example, "pseudo-redshifts" of $\hat{z} \simeq 0.2$ are obtained for both GRBs applying the method of Ref. [33]. It is estimated that this method is accurate to about a factor of 2 , so we take a conservative value of $z=0.1$, bearing in mind that the redshifts may be as high as $z=0.4$. The latter value would improve our sensitivity by about a factor of 4 .

For each of GRB 930131 and GRB 960924, we simulate albedo photons in the relevant energy range, approximating the albedo energy spectra by a polynomial fit to Fig. 5 of Ref. [2]. The degree of linear polarization in the incident radiation is taken as $\Pi=\left\langle s_{1}\right\rangle^{2}+\left\langle s_{2}\right\rangle^{2}$, where $\left\langle s_{j}\right\rangle$ is the average of the photon Stokes parameter $s_{j}$. Drawing from the above distributions at fixed values of $\sigma$ and $\xi$, we generate 10000 linearly polarized photons for each GRB with polarization angle $\psi_{0}$. Using Eq. (1), we calculate the rotation of the Stokes vector during propagation and the resulting polarization when the photon reaches the Earth, and we average over the 10000 photons to obtain $\Pi_{\text {eff }}$ and $\psi_{\text {eff }}$. A more sophisticated analysis using the recorded events and taking into account detector efficiencies could in principle be performed but is unlikely to change significantly the reported results.

For GRB 021206, we estimate and subtract background using all RHESSI events [37] and obtain a fit to the remaining energy spectrum. Using this fit to simulate the burst photons and drawing from the resulting distribution at fixed values of $\sigma$ and $\xi$, we generate a set of linearly polarized photons with polarization angle $\psi_{0}$ and propagate them to the Earth via Eq. (1). To model the detection process, we simulate the scatter angle $\phi$ by drawing from the resulting distribution and using the expression $d S / d \phi \propto[1-\mu \Pi \cos 2(\phi-\psi)]$ for the azimuthal scatter rate, where $\mu \simeq 0.2$ is the detector-dependent modulation factor. The effective photon degree of linear polarization $\Pi_{\text {eff }}$ and the effective polarization angle $\psi_{\text {eff }}$ at the Earth can then be extracted by fitting to the effective azimuthal scatter rate.

Figure 2 displays the resulting $\Pi_{\text {eff }}$ as a function of $\sigma$ and $\xi$ for GRB 930131. The value of $\Pi_{\text {eff }}$ lies above $70 \%$ for $\sigma \ll 10^{-37}$ and around $\xi=2 \psi_{0}$. The latter behavior
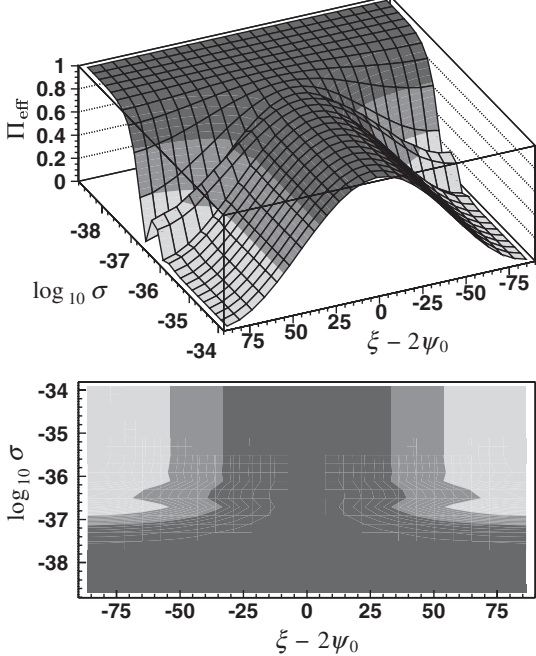

FIG. 2. Effective degree of linear polarization $\Pi_{\text {eff }}$ as a function of $\log _{10} \sigma$ and $\xi$ for GRB 930131. $\Pi_{\mathrm{eff}}=0 \%-35 \%$ (light); $\Pi_{\text {eff }}=35 \%-70 \%$ (gray); $\Pi_{\text {eff }}=70 \%-100 \%$ (dark).

arises because for these values of $\xi$ the Stokes vector $\vec{s}$ is parallel to either $\vec{s}_{+}$or $\vec{s}_{-}$, so polarization measurements are unaffected by relativity violations. The measurement of $\Pi_{930131}>35 \%$ [2] excludes the region extending down to $\log _{10} \sigma<-37$ for this source. Figure 3 shows the results for GRB 960924. A similar result is obtained: the measurement of $\Pi_{930131}>50 \%$ [2] excludes the region extending down to $\log _{10} \sigma<-37$. Figure 4 displays the result for GRB 021206. We find that the claimed value of $\Pi_{021206}=(80 \pm 20) \%$ [18] excludes a region extending down to $\log _{10} \sigma<-38$.

The analysis presented here demonstrates explicitly that the observation of high degrees of polarization for GRB photons with energies ranging to $1 \mathrm{MeV}$ has the potential to probe the coefficients $\tilde{\kappa}_{e+}$ and $\tilde{\kappa}_{o-}$ at the level of $10^{-38}$.
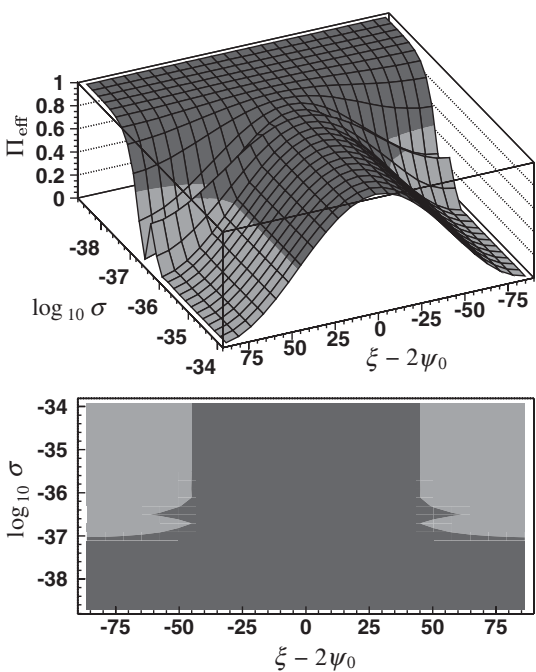

FIG. 3. Effective degree of linear polarization $\Pi_{\text {eff }}$ as a function of $\log _{10} \sigma$ and $\xi$ for GRB 960924. $\Pi_{\text {eff }}=0 \%-50 \%$ (gray); $\Pi_{\text {eff }}=50 \%-100 \%$ (dark). 

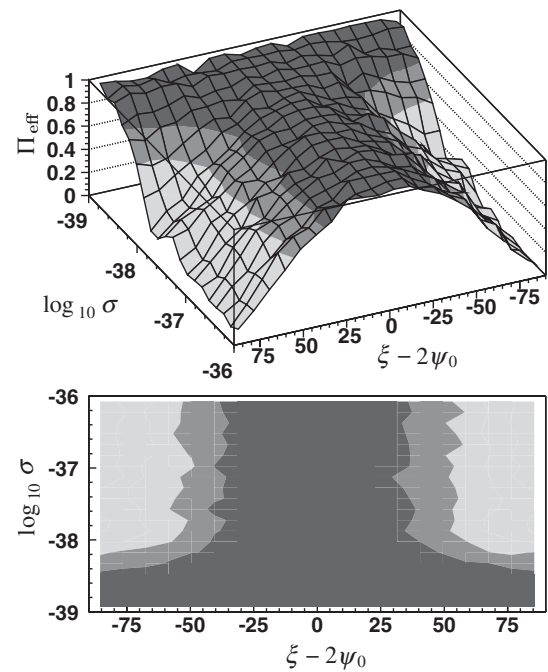

FIG. 4. Effective degree of linear polarization $\Pi_{\text {eff }}$ as a function of $\log _{10} \sigma$ and $\xi$ for GRB 021206. $\Pi_{\text {eff }}=0 \%-60 \%$ (light); $\Pi_{\text {eff }}=60 \%-80 \%$ (gray); $\Pi_{\text {eff }}=80 \%-100 \%$ (dark).

Our results provide the most sensitive test for nonzero $\tilde{\kappa}_{e+}$ and $\tilde{\kappa}_{o-}$ to date, attaining the level of $10^{-37}$. They represent an improvement over existing sensitivities by a conservative factor of 100000 .

This work was supported in part by the United States Department of Energy under Grant No. DE-FG0291ER40661 and by the National Aeronautics and Space Administration under Grant No. NAG3-2194.

[1] For recent theoretical and experimental reviews, see, for example, CPT and Lorentz Symmetry III, edited by V. A. Kostelecký (World Scientific, Singapore, 2005).

[2] D. R. Willis et al., Astron. Astrophys. 439, 245 (2005).

[3] V. A. Kostelecký and M. Mewes, Phys. Rev. Lett. 87, 251304 (2001); Phys. Rev. D 66, 056005 (2002).

[4] D. Colladay and V. A. Kostelecký, Phys. Rev. D 55, 6760 (1997); 58, 116002 (1998).

[5] V. A. Kostelecký, Phys. Rev. D 69, 105009 (2004).

[6] J. A. Lipa, J.A. Nissen, S. Wang, D. A. Stricker, and D. Avaloff, Phys. Rev. Lett. 90, 060403 (2003).

[7] S. Herrmann, A. Senger, E. Kovalchuk, H. Müller, and A. Peters, Phys. Rev. Lett. 95, 150401 (2005); H. Müller, S. Herrmann, C. Braxmaier, S. Schiller, and A. Peters, Phys. Rev. Lett. 91, 020401 (2003).

[8] P. L. Stanwix et al., Phys. Rev. Lett. 95, 040404 (2005); M. E. Tobar, P. Wolf, A. Fowler, and J. G. Hartnett, Phys. Rev. D 71, 025004 (2005); P. Wolf, M. E. Tobar, S. Bize, A. Clairon, A. N. Luiten, and G. Santarelli, Phys. Rev. D 70, 051902 (2004).

[9] P. Antonini, M. Okhapkin, E. Göklü, and S. Schiller, Phys. Rev. A 71, 050101 (2005).

[10] S. M. Carroll, G. B. Field, and R. Jackiw, Phys. Rev. D 41, 1231 (1990).

[11] V. A. Kostelecký and R. Potting, Phys. Rev. D 51, 3923 (1995).
[12] F. Canè et al., Phys. Rev. Lett. 93, 230801 (2004); M. A. Humphrey et al., Phys. Rev. A 68, 063807 (2003); D. Bear et al., Phys. Rev. Lett. 85, 5038 (2000).

[13] P. Wolf et al., Phys. Rev. Lett. 96, 060801 (2006).

[14] B. R. Heckel et al., in CPT and Lorentz Symmetry II, edited by V. A. Kostelecký (World Scientific, Singapore, 2001).

[15] V. A. Kostelecký and C. D. Lane, Phys. Rev. D 60, 116010 (1999).

[16] H. Nguyen et al. (KTeV Collaboration), in CPT and Lorentz Symmetry II (Ref. [14]); R. Ackerstaff et al. (OPAL Collaboration), Z. Phys. C 76, 401 (1997); M. Feindt et al. (DELPHI Collaboration), Report No. DELPHI 97-98 CONF 80, 1997; K. Abe et al. (BELLE Collaboration), Phys. Rev. Lett. 86, 3228 (2001); B. Aubert et al. (BABAR Collaboration), Phys. Rev. Lett. 92, 142002 (2004); J. M. Link et al. (FOCUS Collaboration), Phys. Lett. B 556, 7 (2003); V. A. Kostelecký, Phys. Rev. Lett. 80, 1818 (1998); Phys. Rev. D 61, 016002 (2000); 64, 076001 (2001).

[17] L. B. Auerbach et al. (LSND Collaboration), Phys. Rev. D 72, 076004 (2005); M. D. Messier, in CPT and Lorentz Symmetry III (Ref. [1]); T. Katori et al., hep-ph/0606154; V. A. Kostelecký and M. Mewes, Phys. Rev. D 69, 016005 (2004); 70, 031902(R) (2004); 70, 076002 (2004).

[18] W. Coburn and S.E. Boggs, Nature (London) 423, 415 (2003).

[19] R. E. Rutledge and D. B. Fox, Mon. Not. R. Astron. Soc. 350, 1288 (2004).

[20] S. E. Boggs and W. Coburn, astro-ph/0310515.

[21] C. Wigger, W. Hajdas, K. Arzner, M. Gudel, and A. Zehnder, Astrophys. J. 613, 1088 (2004).

[22] T. Mizuno et al., Nucl. Instrum. Methods Phys. Res., Sect. A 540, 158 (2005).

[23] H. Tajima et al., astro-ph/0407114.

[24] N. Produit et al., Nucl. Instrum. Methods Phys. Res., Sect. A 550, 616 (2005).

[25] E. Costa et al., Nuovo Cimento Soc. Ital. Fis. C 28, 853 (2005).

[26] Q. G. Bailey and V. A. Kostelecký, Phys. Rev. D 74, 045001 (2006); V. A. Kostelecký and S. Samuel, Phys. Rev. Lett. 63, 224 (1989); Phys. Rev. D 40, 1886 (1989).

[27] V. A. Kostelecký and S. Samuel, Phys. Rev. D 39, 683 (1989); V. A. Kostelecký and R. Potting, Nucl. Phys. B359, 545 (1991).

[28] R. Bluhm and V. A. Kostelecký, Phys. Rev. D 71, 065008 (2005); B. Altschul and V. A. Kostelecký, Phys. Lett. B 628, 106 (2005).

[29] V. A. Kostelecký and R. Potting, Gen. Relativ. Gravit. 37, 1675 (2005).

[30] I. G. Mitrofanov, Nature (London) 426, 139 (2003).

[31] T. A. Jacobson, S. Liberati, D. Mattingly, and F.W. Stecker, Phys. Rev. Lett. 93, 021101 (2004).

[32] S.E. Boggs, C. B. Wunderer, K. Hurley, and W. Coburn, Astrophys. J. 611, L77 (2004).

[33] J.-L. Atteia, Astron. Astrophys. 407, L1 (2003).

[34] E. E. Fenimore and E. Ramirez-Ruiz, astro-ph/0004176.

[35] B. E. Schaefer, M. Deng, and D. L. Band, Astrophys. J. 563, L123 (2001).

[36] J. P. Norris, G. F. Marani, and J. T. Bonnell, Astrophys. J. 534, 248 (2000).

[37] HEDC-HESSI Experimental Data Center, ETH Zurich, www.hedc.ethz.ch (August 21, 2001). 